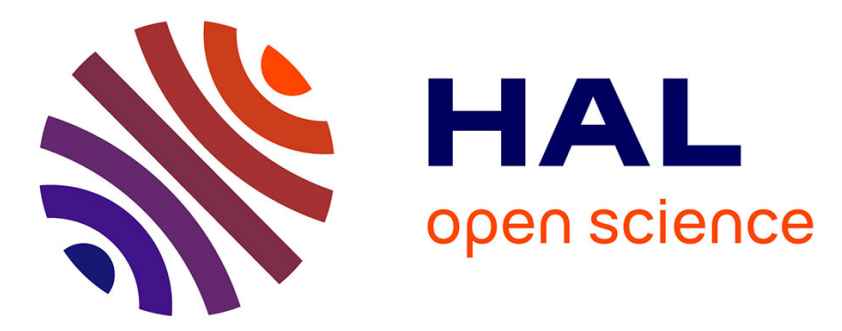

\title{
A fresh look at dense hydrogen under pressure. IV. Two structural models on the road from paired to monatomic hydrogen, via a possible non-crystalline phase
}

Vanessa Labet, Roald Hoffmann, N W Ashcroft

\section{- To cite this version:}

Vanessa Labet, Roald Hoffmann, N W Ashcroft. A fresh look at dense hydrogen under pressure. IV. Two structural models on the road from paired to monatomic hydrogen, via a possible non-crystalline phase. Journal of Chemical Physics, 2012, 136, 10.1063/1.3679751 . hal-02202234

\author{
HAL Id: hal-02202234 \\ https://hal.science/hal-02202234
}

Submitted on 31 Jul 2019

HAL is a multi-disciplinary open access archive for the deposit and dissemination of scientific research documents, whether they are published or not. The documents may come from teaching and research institutions in France or abroad, or from public or private research centers.
L'archive ouverte pluridisciplinaire HAL, est destinée au dépôt et à la diffusion de documents scientifiques de niveau recherche, publiés ou non, émanant des établissements d'enseignement et de recherche français ou étrangers, des laboratoires publics ou privés. 


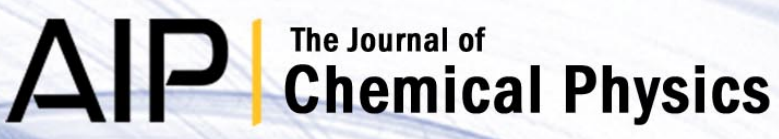

A fresh look at dense hydrogen under pressure. IV. Two structural models on the road from paired to monatomic hydrogen, via a possible noncrystalline phase

Vanessa Labet, Roald Hoffmann, and N. W. Ashcroft

Citation: J. Chem. Phys. 136, 074504 (2012); doi: 10.1063/1.3679751

View online: http://dx.doi.org/10.1063/1.3679751

View Table of Contents: http://jcp.aip.org/resource/1/JCPSA6/v136/i7

Published by the American Institute of Physics.

Additional information on J. Chem. Phys.

Journal Homepage: http://jcp.aip.org/

Journal Information: http://jcp.aip.org/about/about_the_journal

Top downloads: http://jcp.aip.org/features/most_downloaded

Information for Authors: http://jcp.aip.org/authors

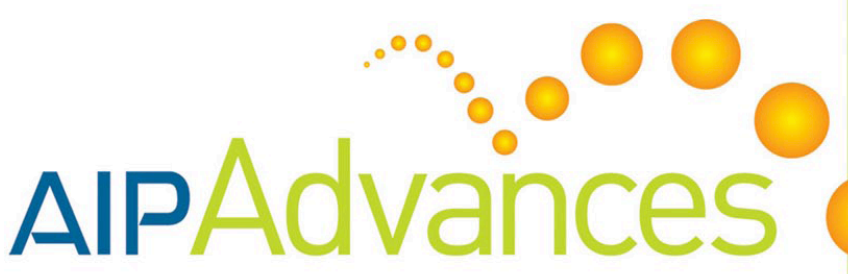

Submit Now
Explore AIP's new open-access journal

Article-level metrics now available

Join the conversation! Rate $\&$ comment on articles 


\title{
A fresh look at dense hydrogen under pressure. IV. Two structural models on the road from paired to monatomic hydrogen, via a possible non-crystalline phase
}

\author{
Vanessa Labet, ${ }^{1, a)}$ Roald Hoffmann, ${ }^{1, b)}$ and N. W. Ashcroft ${ }^{2}$ \\ ${ }^{1}$ Department of Chemistry and Chemical Biology, Cornell University, Baker Laboratory, Ithaca, \\ New York 14853, USA \\ ${ }^{2}$ Laboratory of Atomic and Solid State Physics and Cornell Center for Materials Research, Cornell University, \\ Clark Hall, Ithaca, New York 14853, USA
}

(Received 19 September 2011; accepted 6 January 2012; published online 15 February 2012)

\begin{abstract}
In this paper, we examine the transition from a molecular to monatomic solid in hydrogen over a wide pressure range. This is achieved by setting up two models in which a single parameter $\delta$ allows the evolution from a molecular structure to a monatomic one of high coordination. Both models are based on a cubic Bravais lattice with eight atoms in the unit cell; one belongs to space group $\mathrm{Pa} \overline{3}$, the other to space group $R \overline{3} \mathrm{~m}$. In $P a \overline{3}$ one moves from effective 1-coordination, a molecule, to a simple cubic 6-coordinated structure but through a very special point (the golden mean is involved) of 7-coordination. In $R \overline{3} \mathrm{~m}$, the evolution is from 1 to 4 and then to 3 to 6 -coordinate. If one studies the enthalpy as a function of pressure as these two structures evolve ( $\delta$ increases), one sees the expected stabilization of minima with increased coordination (moving from 1 to 6 to 7 in the $P a \overline{3}$ structure, for instance). Interestingly, at some specific pressures, there are in both structures relatively large regions of phase space where the enthalpy remains roughly the same. Although the structures studied are always higher in enthalpy than the computationally best structures for solid hydrogen those emerging from the Pickard and Needs or McMahon and Ceperley numerical laboratories - this result is suggestive of the possibility of a microscopically non-crystalline or "soft" phase of hydrogen at elevated pressures, one in which there is a substantial range of roughly equi-enthalpic geometries available to the system. A scaling argument for potential dynamic stabilization of such a phase is presented. () 2012 American Institute of Physics. [http://dx.doi.org/10.1063/1.3679751]
\end{abstract}

\section{INTRODUCTION}

At low temperatures and at one atmosphere hydrogen is well known to be a paired molecular solid: each proton has but a single closest neighbor. ${ }^{1-4}$ As pressure increases, however, dense hydrogen is expected to transform eventually into a monatomic system, where protons have more than one closest neighbor, distinct molecules no longer being recognizable in its structure. ${ }^{5}$ The simplest pathway that one might imagine to transform a molecular structure for solid hydrogen to a monatomic structure is a progressive equalization of the shortest and second shortest proton-proton separations which (as discussed earlier in our papers, and with full awareness of the limitations of using molecular language as molecules merge into an extended, delocalized structure) we will refer to as the intramolecular and shortest intermolecular H-H separations, respectively.

In the states of dense hydrogen where pairing is enthalpically preferred, this relatively short range correlation is associated with a prominent intramolecular proton-proton excitation referred to as the vibron or proton-proton stretching mode. Experimentally, the vibron is probed both by Raman scattering and IR spectroscopies. ${ }^{6}$ A major point in what fol-

\footnotetext{
a) Present address: LADIR (UMR 7075 CNRS/UPMC), Université Pierre et Marie Curie, 4 Place Jussieu, 75252 Paris Cedex 05, France.

b) Author to whom correspondence should be addressed. Electronic mail: rh34@cornell.edu.
}

lows is that as a function of relative density increase, the ensuing vibron band is remarkably persistent; Loubeyre et al. ${ }^{7}$ among others have shown that even at 12.7 -fold increase in density - corresponding to $P=316 \mathrm{GPa}$ - the vibron centroid is still observed. It follows that the ordering we choose to call "pairing" of protons remains very much in play under these conditions. Whether we are entitled to refer to the concomitant presence of a bond and a distinct molecule at such notably high average densities is a question we have commented on at some length in the preceding papers, and one we shall again be taking up below.

In the first paper of this series, ${ }^{2}$ we have seen that in some candidate structures for crystalline hydrogen recently proposed in the theoretical study by Pickard and Needs ${ }^{8}$ (and which we have used as a kind of numerical laboratory for learning more about the behavior of dense hydrogen), the shortest intermolecular $\mathrm{H}-\mathrm{H}$ separation decreases as pressure increases, while correspondingly the intramolecular H-H separation lengthens. We introduced in the same paper a convenient measure of this behavior, namely, an equalization function $\xi$ with a domain $[0,1]$ and defined as

$$
\xi(P)=1-\frac{R_{\mathrm{H}_{2}-\mathrm{H}_{2}}(P)-r_{\mathrm{H}-\mathrm{H}}(P)}{R_{\mathrm{H}_{2}-\mathrm{H}_{2}}\left(P_{1 \text { atm }}\right)-r_{\mathrm{H}-\mathrm{H}}\left(P_{1 \mathrm{~atm}}\right)},
$$

with $r_{\mathrm{H}-\mathrm{H}}(P)$ and $R_{\mathrm{H}_{2}-\mathrm{H}_{2}}(P)$ the intramolecular and shortest intermolecular $\mathrm{H}-\mathrm{H}$ separations at pressure $P$, respectively, and $r_{\mathrm{H}-\mathrm{H}}\left(P_{1 \mathrm{~atm}}\right)$ and $R_{\mathrm{H}_{2}-\mathrm{H}_{2}}\left(P_{1 \mathrm{~atm}}\right)$ the intramolecular and 
shortest intermolecular $\mathrm{H}-\mathrm{H}$ separations at $P=1 \mathrm{~atm}$. From its definition $\xi$ takes on value between 0 and $1, \xi=0$ corresponding to the $P=1 \mathrm{~atm}$ case, while $\xi=1$ corresponds to perfect equalization of the intramolecular and shortest intermolecular H-H separations. Following the evolution of this equalization in our numerical laboratories it appears that hydrogen seems to resist perfect equalization of its H-H separations. Or, to put it in other words, that equalization of the separations actually lags behind metallization.

To gain further complementary information, the present paper examines this equalization problem from a different perspective. Probing the possibility (or willingness, or even reluctance) of crystalline hydrogen to move towards an equalization of its intramolecular and shortest intermolecular $\mathrm{H}-\mathrm{H}$ separations under pressure, it will be informative to look at structures, be they perhaps hypothetical, that have a quite special feature, namely, they permit continuous transformation of a paired-phase into a monatomic one, but through the evolution of a single structural parameter. Two space-groups come to mind for elucidating such a process, these being $P a \overline{3}$ and $R \overline{3} \mathrm{~m}$. In this paper, we propose to examine them in detail as numerical paradigms for the study of the systematic equalization of $\mathrm{H}-\mathrm{H}$ separations. ${ }^{9}$

Throughout the following, when we use the word "hydrogen," we again imply the element, and not whether the protons are pairwise ordered or monatomic. For the most part we shall also be carrying out static lattice calculations; as noted earlier this is an inherent limitation of our approach, for the consequences of proton dynamics may be considerable, as we shall see.

\section{COMPUTATIONAL DETAILS}

The two chosen structures have again been investigated using the Vienna $a b$ initio simulation package (VASP) plane wave algorithm, ${ }^{10-12}$ the Perdew-Burke-Ernzerhof generalized gradient approximation density functional, ${ }^{13,14}$ and the projector augmented-wave method ${ }^{15,16}$ with a pseudopotential characterized by a cut-off radius of $0.8 a_{0}$ - the smallest available in the VASP code - and a cut-off of $2000 \mathrm{eV}$ for the kinetic energy of the plane waves. The k-point sets for the Brillouin-zone sampling were generated via the MonkhorstPack scheme, ${ }^{17}$ with a grid of spacing of $2 \pi \times 0.025 \AA^{-1}$. Again, and as noted, the quantum-mechanical zero-point effects for the protons have not been taken into account in the computations, an issue to which we will return.

A matter of quite general concern in high pressure calculations is the use of a pseudopotential for effective electronion (here electron-proton) interactions. In a recent study, McMahon and Ceperley ${ }^{18}$ compared the energies and electron densities of several structures for hydrogen between $P=500 \mathrm{GPa}$ and $P=5 \mathrm{TPa}$, these computed with two choices of pseudopotential cut-off radius: $0.5 a_{0}$ and $0.125 a_{0}$. They concluded that a cut-off radius of $0.5 a_{0}$ was a reasonable approximation. The value we have used is a little larger, at $0.8 a_{0}$. We do not pretend that the present results are highly reliable from a quantitative perspective; our purpose is more to concentrate on the overall enthalpic and structural evolution of two specific structures and for this purpose we believe that the methodology, if imperfect, is nevertheless adequate. And also that this approach is helpful in permitting us to build our chemical and physical intuition into the qualitative behavior, more generally, of solid hydrogen under steady increase of pressure.

\section{PROGRESSIVE EQUALIZATION OF PROTON-PROTON DISTANCES, AND THE PERSISTENCE OF PAIRING: TWO PERIODIC MODELS}

\section{A. Description of the structures}

We now describe in more detail the two structures studied in this work, namely, $P a \overline{3}$ and $R \overline{3} m$. Consider four $\mathrm{H}-\mathrm{H}$ pairs in a low density cubic structure, the axis of each pair being oriented parallel to the direction of one of four different body diagonals of the cube, as sketched in the upper part of Figure 1. Otherwise, the associated pairs have their centers located at the standard sites of a face-centered-cubic cell.

With this specific orientational ordering, which clearly leads to an overall if partial "orientational average," the ensuing $P a \overline{3}$ structure is the one which actually minimizes the electric quadrupolar energy for a 3D-compact structure. ${ }^{19}$ And this is indeed the structure observed for a solid of
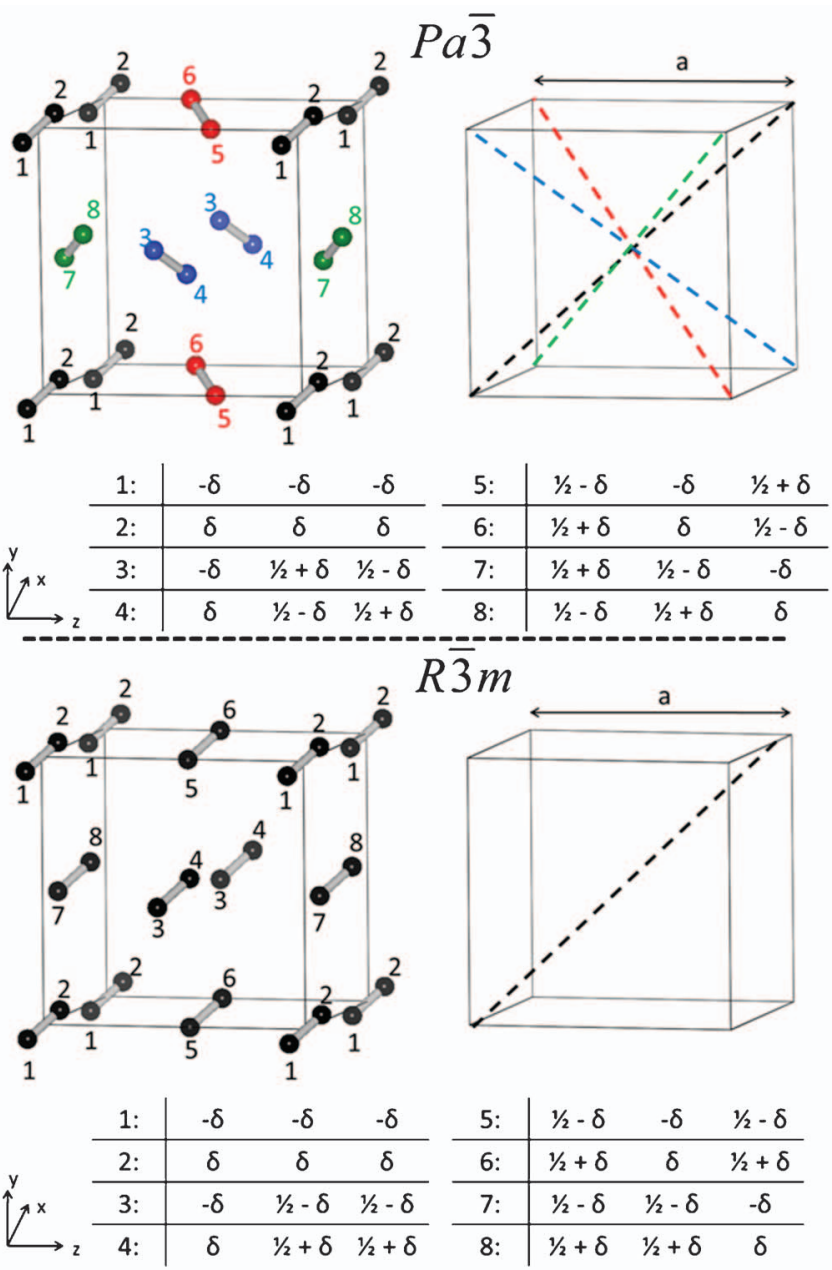

FIG. 1. Unit cells of the $P a \overline{3}$ and $R \overline{3} m$ structures. The coordinates of hydrogen nuclei are given as fractions of the unit cell dimension, $a$. 

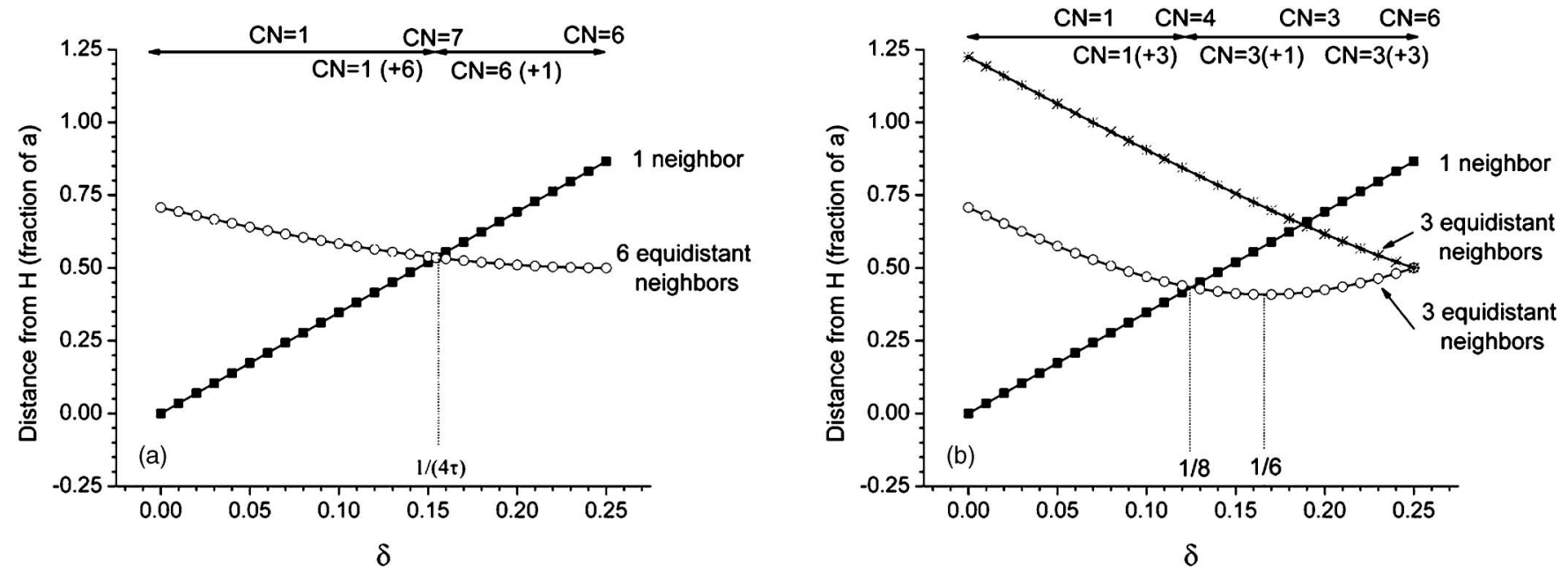

FIG. 2. Evolution of the environment of static protons with $\delta$ in the $P a \overline{3}$ (a) and $R \overline{3} m$ (b) structures. Distances are expressed as fractions of $a$. The open circles are the second nearest proton-proton separations, the filled squares the closest proton-proton separations for small $\delta$. The highly special values of $\delta-\delta=1 /(4 \tau)$ in (a) and $\delta=1 / 8$ and $\delta=1 / 6$ in (b) - are described in the text.

ortho- $\mathrm{H}_{2}$ below $2.8 \mathrm{~K}$ at atmospheric pressure. ${ }^{1}$ It also is the structure of $\alpha$-nitrogen. ${ }^{20}$

Related to $P a \overline{3}$, though not in the angular average sense introduced above, is the "uniaxial" $R \overline{3} m$ structure, represented in the lower part of Figure 1. This is also a cubic structure, and it also involves an 8-hydrogen atom basis. The main difference between the two structures for hydrogen is that in the $R \overline{3} m$ structure all proton-proton pair axes are now oriented in the same direction, along just one of the four body diagonals of the cube (in $P a \overline{3}$ they are sequentially aligned parallel to the four body-diagonals).

\section{B. Evolving coordination numbers}

For both structures, the shortest $\mathrm{H}-\mathrm{H}$ separation is directly related to the value of a single structural parameter, $\delta$, defined in Figure 1. For both structures, and for small $\delta$ values - we will see in a moment what the limit is each proton has one closest neighbor. The key distance, the shortest H-H separation, is then $2 a \delta \sqrt{3}$ where as noted in Fig. $1, a$ is the fundamental cube edge. In the $P a \overline{3}$ structure, each proton then has six second closest neighbors at $a \sqrt{\left(4 \delta^{2}-2 \delta+1 / 2\right)}$ where in the $R \overline{3} m$ structure the second shortest $\mathrm{H}-\mathrm{H}$ separation or shortest intermolecular H-H separation is $2 a \sqrt{\left(3 \delta^{2}-\delta+1 / 8\right)}$ and it involves the proton coordinates of three equi-distant proton pairs. As $\delta$ increases, the intramolecular $\mathrm{H}-\mathrm{H}$ separation increases, whereas the shortest intermolecular $\mathrm{H}-\mathrm{H}$ separation decreases, as shown in Figure 2.

For the $P a \overline{3}$ structure, each proton has but one closest neighbor at a distance of $2 a \delta \sqrt{3}$ so long as $\delta$ is smaller than $1 / 4 \tau$, where $\tau=(1+\sqrt{5} / 2) \approx 1.618 \ldots$. This especial value is the golden mean. For $\delta=1 / 4 \tau$, each proton now has 7 closest neighbors, at a separation of $a \sqrt{3} / 2 \tau \approx 0.535 a$. For $1 / 4 \tau<\delta<1 / 4$ each proton now becomes 6-fold coordinated, with all of the 6 neighbors being at a separation of $a \sqrt{\left(4 \delta^{2}-2 \delta+1 / 2\right)}$. Accordingly, at the particular value $\delta=1 / 4$ the structure is just simple cubic. But for $\delta<1 / 4 \tau$ it can be described as proton-paired, or perhaps even molecular, and is characterized by a value of the equalization function ${ }^{2}$ (Eq. (1)) smaller than 1 . In quite some contrast to this, for $1 / 4 \tau$ $\leq \delta \leq 1 / 4$, the structure can be described as monatomic. The special cases, $\delta=1 / 4 \tau$ and $\delta=1 / 4$ are depicted in Figure 3 .

We hope the reader may now share our appreciation of the wondrous peculiarity of the $P a \overline{3}$ structure for this very particular choice $\delta=1 / 4 \tau$. Note again the especial appearance of the essential quantity, the golden mean (or golden section) $\tau$, which has a way of popping up in the most unexpected geometrical places. ${ }^{22}$ At this precise value of $\delta$, space is now filled (though perhaps not very efficiently) by protons with identical environments (or atoms more generally) that are sevenfold (or hepta-) coordinated, all with identical separations. The arrangement is one of the un-dense sphere packings, and is called "svn" in O'Keeffe et al.'s database of crystal nets. ${ }^{26}$

The local coordination geometry can be described as partway between an axially capped octahedron (along $\overline{3}$ in

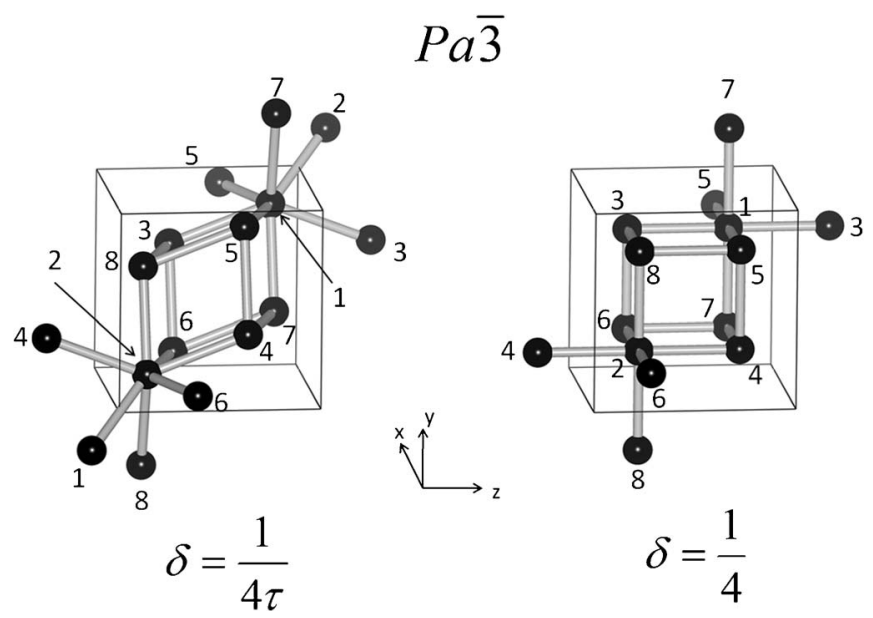

FIG. 3. The $P a \overline{3}$ structure in the particular cases $\delta=1 / 4 \tau$ (7-coordinate) and $\delta=1 / 4$ (6-coordinate, simple cubic) with $(\delta, \delta, \delta)$ the fractional coordinates of proton 2. Here, $\tau$ is the golden mean, or the divine proportion of Pacioli. ${ }^{21-25}$ 


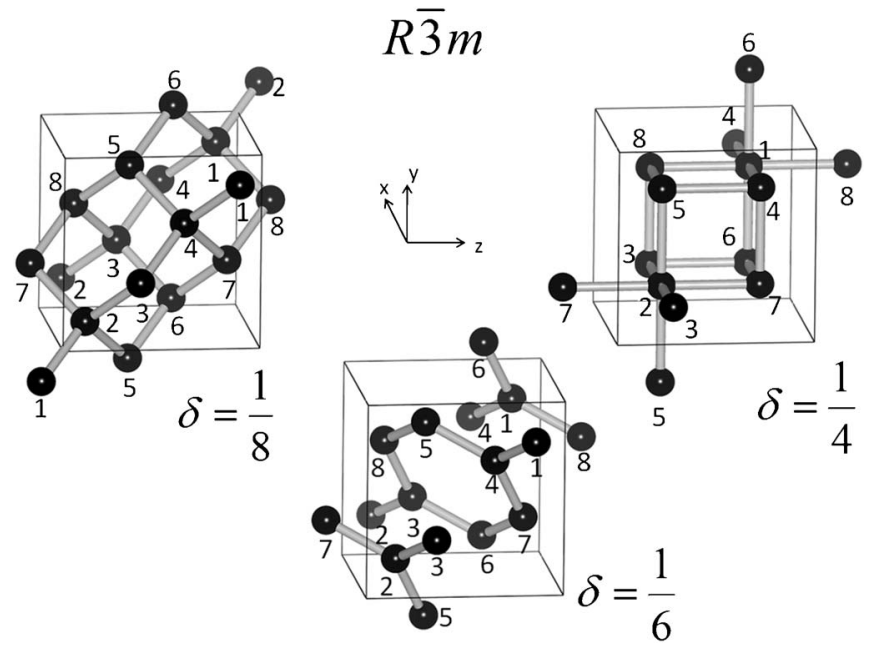

FIG. 4. The $R \overline{3} m$ structure in the particular cases $\delta=1 / 8$ (4-coordinate, cubic diamond), $\delta=1 / 6$ (3-coordinate), and $\delta=1 / 4$ (6-coordinate, simple cubic), with $(\delta, \delta, \delta)$ the fractional coordinates of proton 2.

$P a \overline{3})$ and a trigonal prism. Nothing like this coordination is known in a discrete homonuclear molecular system. ${ }^{27}$ However, the binary system FeSi does crystallize in such a structure with $\delta_{\mathrm{Si}}=0.156$, this being quite close to the ideal value of $1 / 4 \tau \approx 0.154$.

For the $R \overline{3} m$ structure, and for a $\delta$ value smaller than $1 / 8$, each proton now has a single closest neighbor at a separation of $2 a \delta \sqrt{3}$. For $\delta=1 / 8$, the structure is that of cubic diamond and each proton has four closest neighbors at a separation of $a \sqrt{3} / 4 \approx 0.433 a$. In the range $1 / 8<\delta<1 / 4$ each proton now becomes 3-fold coordinated, each of the 3 neighbors being $2 a \sqrt{\left(3 \delta^{2}-\delta+1 / 8\right)}$ away. At the particular value $\delta=1 / 6$ each proton and its three closest neighbors are actually coplanar. To some extent the structure is then quite similar to that of graphite, except for the too small interplanar spacing - the ratio of the next-nearest to nearest interproton distance is $\sqrt{ } 2=1.414 \ldots$ here, but in graphite is 2.35 . For $\delta$ $=1 / 4$ the coordination number of protons increases to 6 and the structure is simple cubic, as is the case for the underlying Bravais lattice of the $P a \overline{3}$ structure. Thus, for $\delta<1 / 8$ the structure can be described as proton-paired, or again perhaps as molecular, but it is monatomic for $1 / 8 \leq \delta \leq 1 / 4$. Structural realizations of the particular cases $\delta=1 / 8, \delta=1 / 6$, and $\delta=1 / 4$ are shown in Figure 4.

\section{At which density might the proton pairs seek to dissociate?}

To investigate how in these structures the proton pairs (with their associated electrons) actually will dissociate, a value was sought of the parameter $\delta$ which minimizes the ground-state enthalpy for several pressures $P$ ranging from $P=150 \mathrm{GPa}$ (where we know from experimental work that solid hydrogen is molecular) to $P=3 \mathrm{TPa}$ where, from the recent work by McMahon and Ceperley, ${ }^{18}$ hydrogen is predicted to be monatomic. Technically, for each pressure this has been achieved by performing geometry optimization calculations, which permitted the volume of the unit cell to change (i.e., the cube length $a$ ) while keeping fixed its shape, as well as the positions of its interior protons. And this was repeated for several $\delta$ values ranging from 0.07 - a "small" value leading to high energy structures with very short intramolecular $\mathrm{H}-\mathrm{H}$ separations - to 0.25 .

What extensive functions with dimensions of energy are to be used in our discussion of the geometrical preferences of these structures? As noted in the first paper of this series, the ground state structures we compute are compared to each other, or in their evolution with a geometrical parameter, in their ground states and under isobaric conditions, and enthalpy is clearly the function of physical importance.

The resulting ground state enthalpy profiles for both kinds of structures are plotted, per proton, as functions of $\delta$ in Figures 5 and 6. As expected, as the pressure increases, the system enthalpy increases. To facilitate comparison of these enthalpy profiles, the computed curves for the way the enthalpy varies with $\delta$ for different pressures $P$ are shown on a relative scale, the global minimal enthalpy along the profile for each $P$ being set to a common zero. The corresponding profiles, plotted in an absolute scale, can be found in the supplementary material $(\mathrm{SM})$ to this paper, Figures $\mathrm{S} 1$ and $\mathrm{S} 2{ }^{28}$

In the case of the $P a \overline{3}$ structure, and for $150 \mathrm{GPa}$ $\leq P \leq 750 \mathrm{GPa}$ the enthalpy profiles display two minima: one occurs before the highly special value of $\delta=1 / 4 \tau$ and the second at $\delta=1 / 4$. For $150 \mathrm{GPa} \leq P \leq 350 \mathrm{GPa}$ the minimum at $\delta<1 / 4 \tau$ is actually the global minimum whereas for $500 \mathrm{GPa} \leq P \leq 750 \mathrm{GPa}$ it is the minimum at $\delta=1 / 4$. By extrapolation of the evolution with pressure of the relative enthalpy of both minima, it may be estimated that at slightly below $P=500 \mathrm{GPa}$ the minimum at $\delta=1 / 4$ becomes lower in enthalpy than that at $\delta<1 / 4 \tau$. As for $\delta=1 / 4 \tau$, this special point clearly corresponds to a maximum. In contrast, for $1 \mathrm{TPa} \leq P \leq 3 \mathrm{TPa}$ the enthalpy profiles show only one minimum at $\delta=1 / 4 \tau$, while $\delta=1 / 4$ corresponds to an enthalpy maximum. Observe, however, just how flat the enthalpy profile is at $P=1 \mathrm{TPa}$ (10 Mbar).

To summarize, as the pressure increases, the preferred coordination number of protons also increases from $1(P<500$ $\mathrm{GPa})$ to $6(500 \mathrm{GPa}<P<750 \mathrm{GPa})$ and then to 7 (1 TPa $<P<3 \mathrm{TPa}$ ), all as expected.

A very similar trend is observed in the case of the $R \overline{3} \mathrm{~m}$ structure. For $150 \mathrm{GPa} \leq P \leq 250 \mathrm{GPa}$ the protons are paired and with an enthalpy profile characterized by two minima - a global one at $\delta<1 / 8$ and a local one at $\delta \approx 1 / 6$, and then two maxima at $\delta=1 / 8$ and $\delta=1 / 4$. At $P=350 \mathrm{GPa}$ the minimum at $\delta \approx 1 / 6$ becomes slightly lower in enthalpy than is found at $\delta<1 / 8$. The protons then have 3 closest neighbors in the optimal conformation $(\delta=1 / 8$ and $\delta=1 / 4$ still correspond to maxima). For $500 \mathrm{GPa} \leq P \leq 600 \mathrm{GPa}$ the protons are again 3-fold coordinated with an enthalpy profile still showing two minima - a global one at $\delta<1 / 8$ and a local one at $1 / 6$ $<\delta<1 / 4$, and also two maxima at $1 / 8<\delta<1 / 6$ and $\delta=1 / 4$. And finally for $750 \mathrm{GPa}<P<3 \mathrm{TPa}$, the protons are 6 -fold coordinated with an enthalpy profile showing a global minimum at $\delta=1 / 4$, a local minimum at $\delta=1 / 8$, and a maximum at $\delta=1 / 6$. Thus, in the static approximation and as the pressure increases, the coordination number increases, now from 1 to 3 , and then to 6 . 

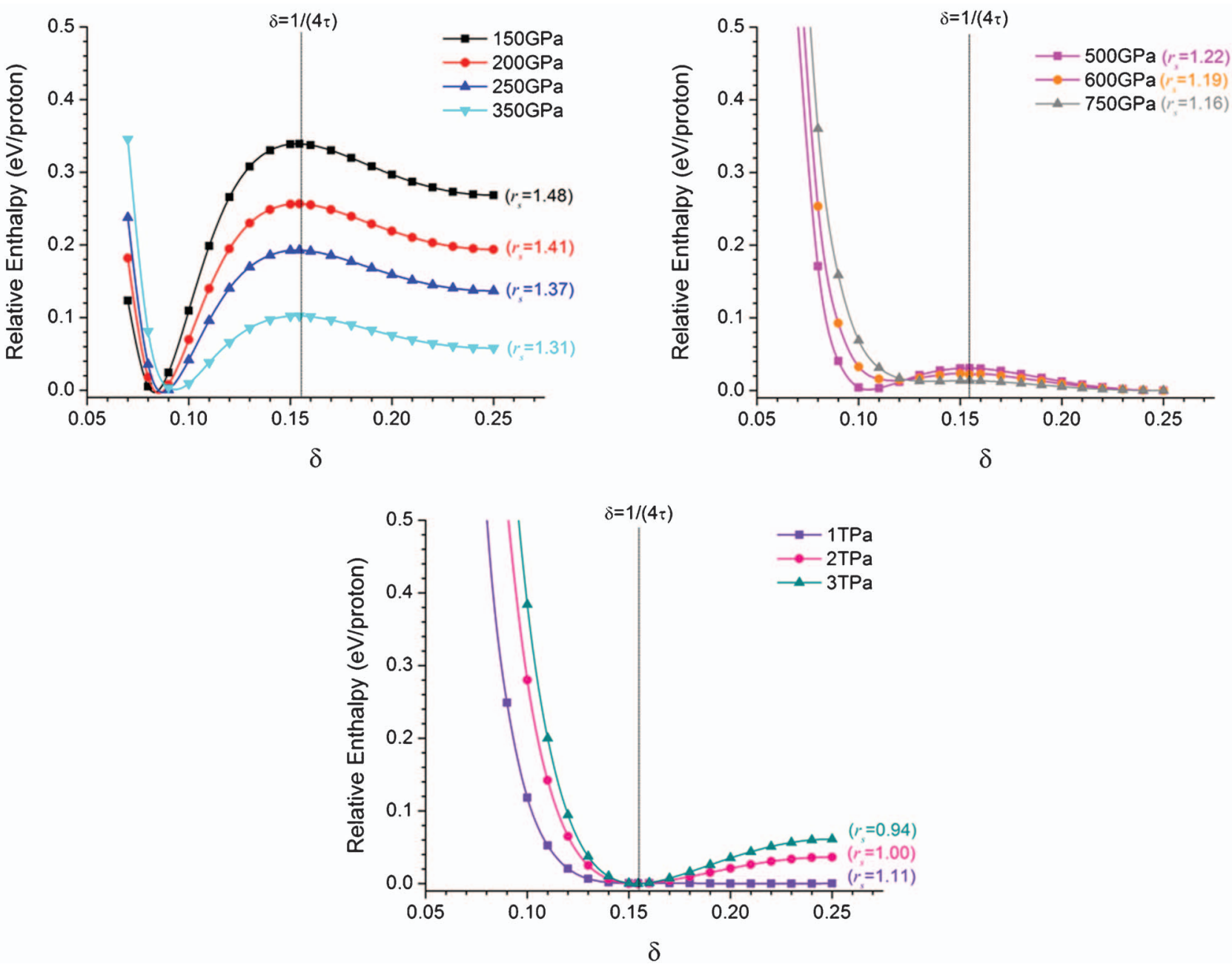

FIG. 5. Relative enthalpies per proton of the $P a \overline{3}$ structures as a function of $\delta$ for several values of pressure $P$. For each curve, the enthalpy of the global minimum is set to zero. The $r_{\mathrm{s}}$ values quoted are those of the structures corresponding to the global minima.

We may note that if protons take up average locations corresponding to these minima, then physically there is expected an associated dynamics both of the centers of masses, and, for a given pair, also of their relative motions (principally the vibron). In addition, however, orientational physics is anticipated, which for low density hydrogen will be revealed in the rotons, these being hindered at higher densities resulting in librational motion. The corresponding internal motions all provide links to possible experimental detection of the transition from paired to monatomic states. A major point is that in the vicinity of such a transition there can be little meaning attached to the descriptors "bond" or "molecule," which were perfectly valid in the original low density initiating system. And the vibrons and rotons will then be subsumed in the phonon modes of the three-dimensional crystal, at least at the level of the harmonic approximation.

\section{A non-crystalline phase?}

In both structures considered above, the transition from paired protons (or molecules) to poly-coordinated protons involves a flattening of the enthalpy profile. In the range $600 \mathrm{GPa} \leq P \leq 1 \mathrm{TPa}$ for $P a \overline{3}$ and $350 \mathrm{GPa} \leq P \leq 600$
GPa for $R \overline{3} m$, dissociation of the proton-pairs costs very little enthalpy. Within a largely static view this therefore raises the possibility that in a macroscopic sample the dissociation may not necessarily proceed in an ordered manner. The corresponding structures might, in fact, be non-crystalline - or amorphous - solids, or indeed even liquids. Our calculations suggest that throughout this pressure range there are no welldefined or preferred intramolecular $\mathrm{H}-\mathrm{H}$ separations. Here we use somewhat tentatively the terminology "soft" to describe this condition of no fixed structure, and characterized by low energy barriers to changes of coordination. Once the transition to equalized distances has been achieved, the energy barriers inducing clear preferred positions of static protons begin to increase again with pressure. The solid then "hardens" or there may even be re-crystallization from a liquid phase.

Nevertheless, given the proximity of enthalpies and the occurrence of unusual coordinations (7-fold, for example) one may now ask about the more general possibility of a noncrystalline state (or states), in proceeding from a paired to a monatomic structure under pressure. A necessary repeat caution is that the quantum-mechanical effects of the proton motion are not included in the methodology used for the present study. As has been highlighted, for example, in the case of 

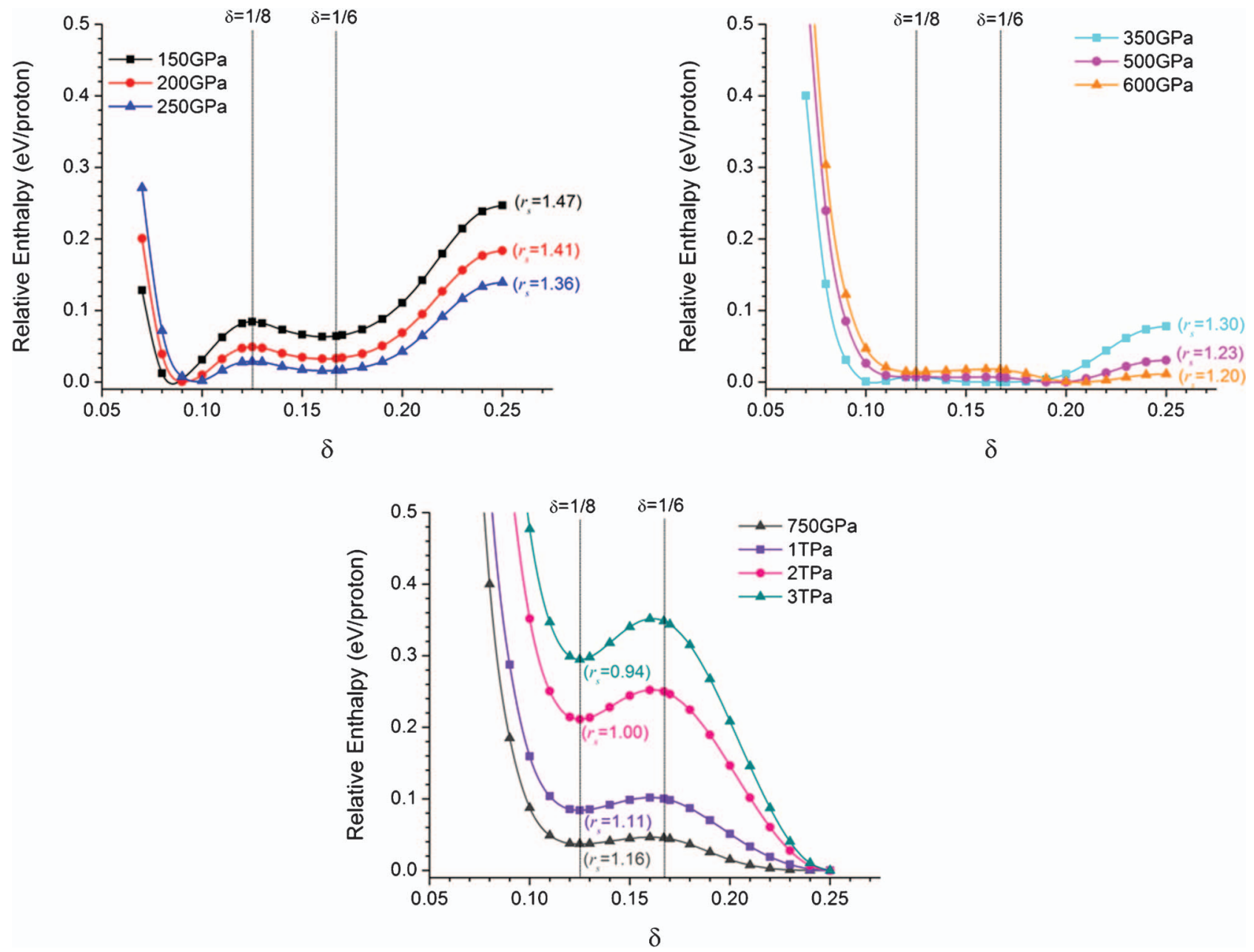

FIG. 6. Relative enthalpies per proton of the $R \overline{3} m$ structures as a function of $\delta$, for several values of pressure $P$. For each curve, the enthalpy of the global minimum is setting the zero. The $r_{\mathrm{s}}$ values quoted are those of the structures corresponding to the global minima.

ice $\mathrm{X}$, the zero-point motion of protons could shift the lowest vibrational level of the proton wave-function above a doublewell barrier. ${ }^{29}$ However, perhaps our observations here will encourage further investigation of hydrogen systems, including dynamical effects explicitly and recognizing once again that a liquid and metallic ground state is also not to be excluded. We present next some simple scaling considerations relevant to this question.

\section{E. A scaling argument}

Having raised the possibility of a departure from crystallinity, an important question arises concerning the likely difference in zero-point energies associated with a dynamic non-diffusing but non-crystalline state (or, to repeat, even a liquid counterpart), and a competing crystalline structure. In the latter, protons are clearly localized in a time average sense, but execute zero-point displacements in a highly phase coordinated way not expected for a liquid, for example. The issue was first raised by London in his arguments in support of the existence of a ground state fluid for ${ }^{4} \mathrm{He} .{ }^{30}$

As is fundamental, the normal modes (or the phonons) of a crystal have frequencies quite characteristic of the un- derlying harmonic oscillator problem, and these are inversely proportional to $\sqrt{m_{p}}$ where $m_{p}$ is the proton mass. In consequence, the zero point energies in the same harmonic approximation are also proportional to $1 / \sqrt{m_{p}}$.

The situation in a system lacking the phase coherence implicit in a crystal is quite different. In a static disordered state, there will be no such phase coordinated displacements, except at very long wavelengths (sound waves will still eventually propagate). Instead, we take it as a property of the microscopically disordered or amorphous state that $N$ protons may indeed be localized near sites $R_{i}$ these now characterizing disorder and again in regions, typified by $2 a_{0} r_{s}$, which may be taken as linear measures of uncertainties in individual displacements. From Heisenberg's principle it must then follow that there is an associated uncertainty in linear momentum of

$$
\sim \frac{\hbar / 2}{2 a_{0} r_{s}} .
$$

And, as with the displacements, it is expected that there is also no coherence in the associated assembly of proton momenta. If $m_{p}$ is the mass of the proton, then the corresponding energy 
must now be

$$
\sim \frac{\left(\frac{\hbar / 2}{2 a_{0} r_{s}}\right)^{2}}{2 m_{p}}
$$

which in Rydbergs (and clearly per proton) is just

$$
\sim \frac{m_{e}}{m_{p}} \frac{3}{8} r_{s}^{2},
$$

and this is typically just a few meV (where $1 \mathrm{meV} \sim 11 \mathrm{~K}$ ).

Though the numerical factors may not be quite secure, observe that the mass dependence is now proportional to $1 / m_{p}$; for the assembly of oscillators the dependence, as above, is as $1 / \sqrt{m_{p}}$. This argument leads to the conclusion that for collective motions constituting individual displacements that are not neighbor-to-neighbor correlated and coherent, the zero point energy per proton can be considerably less than for the coherent collective oscillator (the phonon case). The argument ${ }^{30}$ is crucially dependent on a physical assumption that in a disordered phase the notion of a localization distance can actually arise in the first place. It is obviously a highly self-consistent proposition (for a localized particle must clearly also be a bounding neighbor).

Recalling that the ground state enthalpy necessarily includes proton zero-point energy effects, the question that arises is whether a phase can emerge which, in giving the lowest overall enthalpy at a given pressure, is now determined at least in part by the choice of an arrangement that has the lowest zero-point energy? (We should also recall that the electronic energies for the non-crystalline phase must also enter into the eventual comparison.) The point is a general one and not limited to hydrogen (indeed the argument is a variant of one introduced by London, ${ }^{30}$ as noted above), but is of especial interest here because the enthalpy differences at a given pressure between static phases in this system constituted by fundamentally long-range proton-proton and electron-electron interactions are themselves so small (see the enthalpy curves in the original Pickard and Needs paper ${ }^{8}$ ). Again, this is a matter that can be investigated in part by further consideration of the two structures introduced above. But while a static disordered phase may not be ruled out, it is apparent that the argument applies equally well to a dynamically disordered phase, one which is, however, translationally invariant; in short a ground state fluid. In this instance one is imagining a quantum fluid of protons (or even of deuterons) but which has a quite unique character, namely, that it is metallic.

\section{F. Relative enthalpy of the $P a \overline{3}$ and $R \overline{3} m$ structures with respect to other candidates}

The particular cases of the $P a \overline{3}$ and $R \overline{3} m$ structures suggest the possible occurrence of a non-crystalline solid state as the intramolecular and shortest intermolecular $\mathrm{H}-\mathrm{H}$ separations become equalized in solid hydrogen, this under the effect of a steady increase of pressure. It is obviously difficult, based on just two examples, and lacking a detailed associated analysis of proton dynamics, to determine if the possibility of this suggested non-crystalline state is general. We can ex-

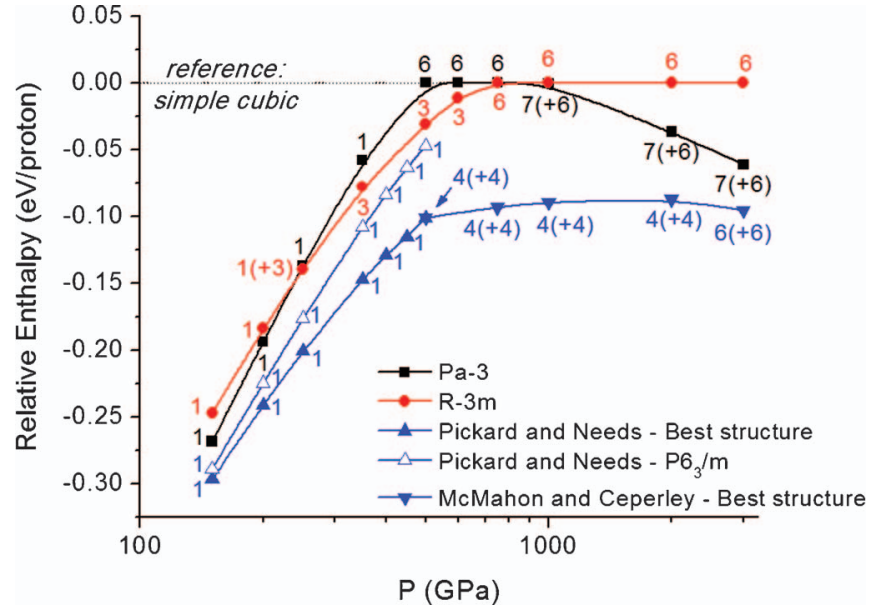

FIG. 7. Relative enthalpies per proton, with respect to the simple cubic structure, of the $\operatorname{Pa} \overline{3}$ and $R \overline{3} m$ structures with their optimal $\delta$ value, of the preferred candidate structures in the Pickard and Needs study, ${ }^{8}$ of the $P 6_{3} / \mathrm{m}$ structure, and of the preferred candidate structures in the McMahon and Ceperley study. ${ }^{18}$ The digits next to the symbols indicate the coordination numbers for the protons.

amine, however, how these two models may fare relative to the other structures we know for dense hydrogen at low temperatures. In the static lattice approximation we compare in Figure 7 the enthalpy differences, as the pressure varies, between the $P a \overline{3}$ and $R \overline{3} m$ structures in their optimal $\delta$ configuration and also the most competitive structures proposed by Pickard and Needs in their recent theoretical study of the hydrogen phase diagram in its ground state and for $P$ $<500 \mathrm{GPa}$ (Ref. 8) $\left(\mathrm{Pb}_{3} / \mathrm{m}\right.$ for $P<105 \mathrm{GPa} ; \mathrm{C} 2 / c$ for 105 $\mathrm{GPa}<P<270 \mathrm{GPa}$; Cmca-12 for $270 \mathrm{GPa}<P<385 \mathrm{GPa}$; Cmca for $385 \mathrm{GPa}<P<490 \mathrm{GPa} ; I_{1} /$ amd for $P>490 \mathrm{GPa}$ when zero-point energies are not included). These may also be compared with those proposed by McMahon and Ceperley ${ }^{18}$ in their recent ground state study of atomic hydrogen for $500 \mathrm{GPa}<P<5 \mathrm{TPa}\left(\right.$ I $_{1}$ /amd for $500 \mathrm{GPa}<P<2.5 \mathrm{TPa}$; a structure of $R \overline{3} m$ symmetry for $2.5 \mathrm{TPa}<P<5 \mathrm{TPa}$, that we will call $R \overline{3} m(2)$ to avoid confusion with the $R \overline{3} m$ structure studied in the present paper (Figure 1), when zero-point energies are not included). A presentation of these latter structures can be found in Figures S7 and S8 of the SM to the present paper. $^{28}$

The enthalpy difference between $P a \overline{3}$ and $R \overline{3} m$ by itself is already interesting. At the lowest pressure considered in the present work, the preferred $P a \overline{3}$ structure is slightly more stable than the lowest $R \overline{3} m$ one. Both structures become of equal enthalpy around $P=250 \mathrm{GPa}$. Then, as the pressure increases, the lowest $R \overline{3} m$ structure becomes more stable than the lowest $P a \overline{3}$ structure until $P=750 \mathrm{GPa}$, where both structures are identical (simple cubic, $\delta=1 / 4$ ). As the pressure goes on decreasing, the best $P a \overline{3}$ structure then becomes the most stable and the difference in enthalpy with the best $R \overline{3} m$ structure increases as the pressure increases.

This ordering can be readily rationalized in terms of coordination number considerations. The coordination number of the best $P a \overline{3}$ and $R \overline{3} m$ structures is indicated on Figure 7, close to each representative point. We know from Sec. III B that our $P a \overline{3}$ and $R \overline{3} m$ "transits" sweep through 
$1 \rightarrow 7 \rightarrow 6$ and $1 \rightarrow 4 \rightarrow 3 \rightarrow 6$ coordination as $\delta$ increases for a given volume of the unit cell. Then, the $R \overline{3} \mathrm{~m}$ transit covers a lower coordination range and so at intermediate pressure (approximately to $350 \mathrm{GPa}<P<750 \mathrm{GPa}$ ), this structure, in a tri-coordinate form, is in fact at a lower enthalpy than $P a \overline{3}$. At still higher pressure, where one expects higher coordination, ${ }^{31}$ one enters a region of preference for $P a \overline{3}$ in its unusual hepta-coordinate configuration, the highest coordination achievable by the ensemble of the $P a \overline{3}$ and $R \overline{3} \mathrm{~m}$ structures. In a restricted world where $P a \overline{3}$ and $R \overline{3} m$ would be the only possible structures for crystalline hydrogen, protons would proceed from $1 \rightarrow 3 \rightarrow 6 \rightarrow 7$ coordination under an increase of pressure.

In the Pickard and Needs calculations, ${ }^{8}$ the monatomic structure $\left(I 4_{1} /\right.$ amd $)$, which appears around $P=500 \mathrm{GPa}\left(r_{s}\right.$ $=1.23$ ) is 4 -coordinated at a proton - but really $4+4$ if we define the coordination number based on the maximum gap in the histogram showing the number of neighbors as a function of their distances. Note that a coordination of 4 is achievable in the $R \overline{3} \mathrm{~m}$ structure at the particular value of $\delta$ $=1 / 8$, where it is then identical to the cubic diamond structure. Nonetheless, this $R \overline{3} m$ waypoint was never the lowest enthalpy structure for the particular pressure values chosen in our calculations.

Over the entire range of densities explored in our study, the $P a \overline{3}$ and $R \overline{3} m$ structures are themselves never quite competitive as actual ground state candidate structures, neither with the preferred Pickard and Needs structures (blue curve, full triangles pointing towards the top in Figure 7) nor the preferred McMahon and Ceperley structures (blue curve, full triangles pointing towards the bottom in Figure 7$)$. The difference (by how much the $P a \overline{3}$ and $R \overline{3} m$ structures are less stable) appears to increase as the density increases, up to $500 \mathrm{GPa}$. For instance, at $P \approx 450 \mathrm{GPa}\left(r_{s}=1.25\right)$, the $\mathrm{Cmca}$ structure that Pickard and Needs and others ${ }^{32}$ found to be the most stable for dense, static hydrogen between $P=385 \mathrm{GPa}$ and $P=490 \mathrm{GPa}$ (see supplementary material ${ }^{28}$ ), is about $70 \mathrm{meV} /$ proton more stable than the lowest enthalpy $R \overline{3} \mathrm{~m}$ structure. Even the $P 6_{3} / m$ structure (most stable at low densities, but not favored at high), is at least $20 \mathrm{meV} /$ proton more stable.

Nevertheless, the final word on the stability of the various structures may not be at hand, for these calculations do omit dynamic effects. Indeed, the energy differences between various structures remain notably low with respect to the zeropoint energy, which at $P=500 \mathrm{GPa}\left(r_{s} \sim 1.23\right)$ is estimated at $325 \mathrm{meV} /$ proton in the $\mathrm{Cmca}$ molecular solid, and at $300 \mathrm{meV} /$ proton in the $I_{1} /$ amd monatomic phase. ${ }^{18}$ Between $P=500 \mathrm{GPa}\left(r_{s} \sim 1.23\right)$ and $P=1 \mathrm{TPa}\left(r_{s} \sim 1.11\right)$, the enthalpy gap between the $I 4_{1} /$ amd structure and the best structure among $P a \overline{3}$ and $R \overline{3} m$ remains quite constant. At higher pressure, the gap starts to decrease, suggesting that the $P a \overline{3}$ structure in its hepta-coordinated form could indeed become competitive at higher pressures.

\section{CONCLUDING COMMENTS}

Thus far, so good. We have examined two quite plausible models for the progressive equalization of the intramolecular and shortest intermolecular $\mathrm{H}-\mathrm{H}$ separations, in which a single geometrical parameter allows us to follow closely the paired to unpaired (or molecular to monatomic) continuous transformation. And we obtain the expected progression of stability in these representative models - as pressure increases and $r_{s}$ decreases, the system moves from lower to higher coordination. But, and this is a significant reservation, the $P a \overline{3}$ and $R \overline{3} m$ structures are definitely not the most stable choices in enthalpic terms for static dense hydrogen.

Surely, then, it would be most satisfactory if there were further and perhaps more general space groups in which one could place $(N / 2)$ proton-pairs in positions described by but a single parameter. As above, the notion would be that progressive evolution of this parameter should sweep gradually through 1- to 12-fold coordinations, thus passing through every common coordination number. There is no such space group to date, apparently. The two groups we studied here do move through the coordination sequence $1 \rightarrow 4 \rightarrow 3$ $\rightarrow 6(R \overline{3} m)$ and $1 \rightarrow 7 \rightarrow 6(P a \overline{3})$, changing by a continuous transformation from an initially paired, molecular structure into a monatomic one through the evolution of a single structural parameter.

The associated enthalpics suggest that the possible dissociation of proton-pairs may proceed by virtue of a very flat enthalpy surface, a region of phase space which could therefore even encompass non-crystalline, amorphous, microscopically "soft" or even liquid arrangements. By invoking elementary uncertainty principle arguments (also generalizable to possible fluid phases), such states may indeed be favored upon inclusion of proton dynamics. The generality of this result has yet to be investigated for some more realistic relevant structures for solid hydrogen at the densities involved.

\section{GENERAL CONCLUSION}

Let us now put together what we have learnt about possible equalization of the $\mathrm{H}-\mathrm{H}$ separations in dense hydrogen in the four papers of this series. We started this study ${ }^{2}$ with a structural analysis of some static candidate structures for solid hydrogen recently proposed by Pickard and Needs in their theoretical study of the phase diagram of element 1 in its ground state and for pressures $P<500 \mathrm{GPa} .{ }^{8}$ These structures, limited as they are in insufficiently accounting for rotation and libration, nevertheless provide us with a numerical laboratory for exploring the primary physical factors operative in the approach to metallization.

Following then the most stable structures, we have examined the evolution of the intramolecular and shortest intermolecular $\mathrm{H}-\mathrm{H}$ separations as the pressure varies. As in the entire series of papers, we use molecular language here, quite cognizant of its ultimate limitations as the dense regime is entered. However, in agreement with general intuition, the shortest intermolecular H-H separation decreases as the pressure increases; first dramatically and then more progressively. The intramolecular $\mathrm{H}-\mathrm{H}$ separations dance to a different tune. As the pressure is increased, the intramolecular $\mathrm{H}-\mathrm{H}$ separation first shortens $(P<100 \mathrm{GPa})$, then lengthens $(100 \mathrm{GPa}$ $<P<400 \mathrm{GPa}$ ), and at still higher pressure (400 GPa $<P$ $<500 \mathrm{GPa}$ ) shortens again. The changes are never dramatic; 
the intramolecular $\mathrm{H}-\mathrm{H}$ separation remains remarkably constant, in fact close to $0.75 \AA$. We defined an index to measure the degree of equalization of the intramolecular and shortest intermolecular $\mathrm{H}-\mathrm{H}$ separations as a function of pressure; this index particularly highlights the fact that hydrogen seems to resist complete equalization of its $\mathrm{H}-\mathrm{H}$ distances.

Then, in the second paper of this series, ${ }^{3}$ we focused on the evolution of the intramolecular $\mathrm{H}-\mathrm{H}$ separation as the pressure increases, attempting to understand both its nonmonotonous behavior, and the small range of its excursions. In this task, discrete molecular models for the progression under pressure assisted - some of them already present in the literature in the earlier work of Herschbach and collaborators. We proposed that the shortening of the intramolecular $\mathrm{H}-\mathrm{H}$ separation, concomitant with a stiffening of the bond, arises from spatial confinement of the $\mathrm{H}_{2}$ molecules as neighboring $\mathrm{H}_{2}$ units steadily encroach. As for the lengthening and weakening of the intramolecular $\mathrm{H}-\mathrm{H}$ separation, we found its origins to be in orbital interactions, especially in the interaction and mixing of occupied MOs of some $\mathrm{H}_{2}$ units and empty MOs of others.

If the first effect (contraction with increasing pressure) is a clearly physical one, the second effect (elongation of the bond as the density increases) we chose to regard as more chemical in origin, and we have related it to the observed elongation of the $\mathrm{H}-\mathrm{H}$ bond in some known and beautiful molecular dihydrogen complexes.

In the third paper of the series, ${ }^{4}$ we examined further these arguments but in the context of the candidate structures for solid hydrogen studied in the first paper. Fully aware of the approximation implicit in proposing molecular units (fragments) in the dense solid, we performed a fragment molecular orbital analysis of these structures. They showed clearly that the bonding MOs of the $\mathrm{H}_{2}$ units depopulate under pressure, while the antibonding counterparts gain electrons. Another molecular model gave us a clear estimate of how much bond lengthening should be associated with a given orbital population. Returning to the extended solid, for the calculated extent of electron transfer (from bonding to antibonding orbitals) in that solid, one would expect far more elongation of the intramolecular H-H separation than is in fact observed.

Clearly, two opposing effects are at work. Spatial confinement that shortens and stiffens the intramolecular $\mathrm{H}-\mathrm{H}$ bonds actually coexists (and competes) with orbital interactions that have the opposite effect.

In the present fourth and last paper, we sought complementary and largely geometric information about the possible equalization of the $\mathrm{H}-\mathrm{H}$ separations in crystalline structures for static hydrogen by studying two hypothetical extended structures which possess the advantage of permitting continuous transformation from molecular states to monatomic ones through the evolution of but a single structural parameter. As in the three first papers, we followed the intra- and shortest intermolecular H-H separations as the pressure increases. But we also paid particular attention to the evolution of the enthalpy surface as the pressure increases. It appears that at intermediate pressures, between a regime where paired states (molecules) are highly preferred over unpaired, and a high density one where the opposite is true, the enthalpy sur- face associated with the in-phase stretching mode of the $\mathrm{H}_{2}$ molecules becomes remarkably flat. This relatively extensive region of phase space where a large range of $\mathrm{H}-\mathrm{H}$ separations are roughly equi-enthalpic is suggestive of dissociation of the $\mathrm{H}_{2}$ units via a non-crystalline, amorphous, microscopically plastic or soft state, or possibly even a liquid. To examine just how general this behavior is, it will be essential in the future to include dynamical effects in the theoretical studies of dense hydrogen.

A natural question then finally arises: how, experimentally, will it be possible to confirm or exclude the existence of such a non-crystalline state or states? As the pressure increases, the electronic distribution in hydrogen is expected to resemble more and more that of a non-interacting electron gas, seeded by nuclei, to be sure. But a uniform free electron gas does not scatter x-rays in any structurally revealing way. Thus, there is probably not much to expect from x-ray diffraction experiments once they will be become feasible - no doubt this day will come soon. Neutron diffraction experiments will probably be more revealing. Probing the H-H vibron by infrared and Raman spectroscopy will surely be essential and the evolution of the IR intensity, in particular, will be interesting. If hydrogen does adopt the Cmca structure at $\sim 400 \mathrm{GPa}$, as has been suggested, ${ }^{32,8}$ an eventual decrease of the infrared activity is then expected.

We trust we have justified the title of our series of papers - here is a fresh look at an old problem of continuing importance.

\section{ACKNOWLEDGMENTS}

We thank Michael O'Keeffe and Paul Loubeyre for illuminating discussions. Our work was supported by EFree, an Energy Frontier Research Center funded by the U.S. Department of Energy (DOE), Office of Science, Office of Basic Energy Sciences under Award No. DESC0001057 at Cornell. The work was also funded by the National Science Foundation (NSF) through (Grant Nos. CHE-0910623 and Grant DMR-0907425). V.L. would like to thank the FrancoAmerican Commission for Educational Exchange for its financial support. Calculations were performed in part at the Cornell NanoScale Facility, a member of the National Nanotechnology Infrastructure Network, which is supported by the NSF (Grant No. ECS-0335765). This research was also supported by the NSF through TeraGrid resources provided by NCSA.

${ }^{1}$ J. Van Kranendonk, Solid Hydrogen (Plenum, New York, 1983).

${ }^{2}$ V. Labet, P. Gonzalez-Morelos, R. Hoffmann, and N. W. Ashcroft, J. Chem. Phys. 136, 074501 (2012).

${ }^{3}$ V. Labet, R. Hoffmann, and N. W. Ashcroft, J. Chem. Phys. 136, 074502 (2012).

${ }^{4}$ V. Labet, R. Hoffmann, and N. W. Ashcroft, J. Chem. Phys. 136, 074503 (2012).

${ }^{5}$ E. Wigner and H. B. Huntington, J. Chem. Phys. 3, 764 (1935).

${ }^{6}$ H. K. Mao and R. J. Hemley, Rev. Mod. Phys. 66, 671 (1994).

${ }^{7}$ P. Loubeyre, F. Occelli, and R. LeToullec, Nature (London) 46, 613 (2002). ${ }^{8}$ C. J. Pickard and R. J. Needs, Nat. Phys. 3, 473 (2007).

${ }^{9}$ For an early molecular model of equalization, see W. T. Borden, Theor. Chim. Acta 69, 171 (1986).

${ }^{10}$ G. Kresse and J. Hafner, Phys. Rev. B 47, 558 (1993).

${ }^{11}$ G. Kresse and J. Furthmüller, Comput. Mater. Sci. 6, 15 (1996). 
${ }^{12}$ G. Kresse and J. Furthmüller, Phys. Rev. B 54, 11169 (1996).

${ }^{13}$ J. P. Perdew, K. Burke, and M. Ernzerhof, Phys. Rev. Lett. 77, 3865 (1996).

${ }^{14}$ J. P. Perdew, K. Burke, and M. Ernzerhof, Phys. Rev. Lett. 78, 1396 (1997).

${ }^{15}$ P. E. Bloch, Phys. Rev. B 50, 17953 (1994).

${ }^{16}$ G. Kresse and D. Joubert, Phys. Rev. B 59, 1758 (1999).

${ }^{17}$ H. J. Monkhorst and J. D. Pack, Phys. Rev. B 13, 5188 (1976).

${ }^{18}$ J. M. McMahon and D. M. Ceperley, Phys. Rev. Lett. 106, 165302 (2011).

${ }^{19}$ J. Felsteiner, Phys. Rev. Lett. 15, 1025 (1965).

${ }^{20}$ L. Vegard, Z. Phys. 58, 497 (1929).

${ }^{21}$ L. Pacioli, De Divina Proportione (Luca Paganinem de Paganinus de Brescia (Antonio Capella), Venice, 1509).

${ }^{22}$ M. Livio, The Golden Ratio: The Story of Phi, the World's Most Astonishing Number (Broadway Books, New York, 2002).

${ }^{23}$ H. E. Huntley, The Divine Proportion: A Study in Mathematical Beauty (Dover, New York, 1970).
${ }^{24} \mathrm{H}$. Walser, The Golden Section (Mathematical Association of America, Washington, D.C., 2001).

${ }^{25}$ R. A. Dunlap, The Golden Ratio and Fibonacci Numbers (World Scientific, Singapore, 1997).

${ }^{26}$ M. O'Keeffe, M. A. Peskov, S. J. Ramsden, and O. M. Yaghi, Accts. Chem. Res. 41, 1782 (2008).

${ }^{27}$ D. Casanova, P. Alemany, J. M. Bofill, and S. Alvarez, Chem. -Eur. J. 9, 1281 (2003)

${ }^{28}$ See supplementary material at http://dx.doi.org/10.1063/1.3679751 for this figure.

${ }^{29}$ M. Benoiy, D. Marx, and M. Parrinello, Nature (London) 392, 258 (1998).

${ }^{30}$ F. London, Phys. Rev. 54, 947 (1938).

${ }^{31}$ W. Grochala, R. Hoffmann, J. Feng, and N. W. Ashcroft, Angew. Chem., Int. Ed. 46, 3620 (2007).

${ }^{32}$ K. A. Johnson and N. W. Ashcroft, Nature (London) 403, 632 (2000) 